that these parameters may be useful to identify patients with gall bladder dyskinesia who will benefit from cholecystectomy.

Competing interests None declared.

\section{PWE-154 PRIMARY DUCT CLOSURE AFTER LAPAROSCOPIC BILE DUCT EXPLORATION FOR CHOLEDOCHOLITHIASIS IS A SAFE AND EFFECTIVE APPROACH}

doi:10.1136/gutjnl-2012-302514d.154

${ }^{1} Y$ Khaled, ${ }^{*}{ }^{1} \mathrm{D} J$ Malde, ${ }^{1,2} \mathrm{~B}$ J Ammori. ${ }^{1}$ General Surgery HPB unit, North Manchester General Hospital, Manchester, UK; ${ }^{2}$ The University of Manchester, Manchester, UK

Introduction The common bile duct is traditionally managed with Ttube drainage after choledochotomy and removal of common bile duct (CBD) stones, but this approach carries an associated tuberelated morbidity rate, including bile leak, of $10.5-20 \%$. This study examines the safety and effectiveness of laparoscopic CBD exploration (LCBDE) followed by primary duct closure.

Methods This is a retrospective analysis of 94 consecutive patients (27 male) who underwent LCBDE between October 2002 and December 2011. The duct was primarily closed in all patients. The results shown represent the median (range).

Results All procedures were completed laparoscopically. The maximum diameter of the CBD was $9.7(3-30) \mathrm{mm}$, and it was dilated in $93 \%$ of patients. The number of CBD stones was $2(0-20)$. The exploration was transcystic in 14 patients and trans-CBD in 80 patients. The biliary tree was clear at the end of exploration with no subsequent evidence of retained stones in 92 patients (97.8\%). The operating time was $117(22-395) \mathrm{min}$. Postoperative bile leak occurred in four patients (4.5\%) who were managed successfully with re-laparoscopy and suturing of the choledochotomy $(n=2)$, laparoscopic insertion of biliary stent $(n=1)$ and conservatively $(n=1)$. The overall morbidity rate was $8 \%$ and included pulmonary complications $(n=3)$, cholangitis $(n=2)$, myocardial infarction $(n=1)$ and wound infection $(n=1)$. There were no operative deaths, and the postoperative hospital stay was $1(0-51)$ day. At a follow-up of $48.2(24-82)$ months, $92.5 \%$ of patients ( $n=87$ ) had no biliary symptoms, one patient required endoscopic extraction of a retained stone, one developed bile duct stricture that was managed successfully by endoscopic balloon dilatation, and four patients (4.5\%) failed to attend the follow-up.

Conclusion Primary duct closure following LCBDE is safe, and can be employed as an alternative to T-tube insertion with short hospital stay and lower morbidityrate.

Competing interests None declared.

\section{PWE-155 THE SAFETY AND EFFICIENCY OF LAPAROSCOPIC LIVER RESECTION FOR BENIGN AND MALIGNANT LIVER DISEASES}

doi:10.1136/gutjnl-2012-302514d.155

${ }^{1} Y$ S Khaled, ${ }^{*}{ }^{1} \mathrm{D}$ J Malde, ${ }^{1} \mathrm{R}$ Deshpande, ${ }^{1} \mathrm{~N}$ de' Liguori Carino, ${ }^{1,2} \mathrm{~B} \mathrm{~J}$ Ammori. ${ }^{1}$ General Surgery HPB unit, North Manchester General Hospital, Manchester, UK; ${ }^{2} T$ The University of Manchester, Manchester, UK

Introduction Advances in technology and techniques facilitated the development of laparoscopic liver resection (LLR). The study is aimed at the evaluation of the feasibility and effectiveness of LLR for benign and malignant pathology.

Methods This is a retrospective study of 61 patients (27 female) aged 63 (25-83) years who underwent LLR for benign $(n=9)$ and malignant $(n=52)$ between 2003 and 2011 in a single UK tertiary centre. The results shown represent median (range).
Results Surgery was completed laparoscopically in 60 patients (98.3\%) and converted to open due to extensive abdominal adhesions in one patient. The procedures performed included the resection of one segment $(n=16)$, two segments $(n=36)$ and three segments $(n=9)$. The overall operative morbidity was $8.5 \%$ and there was no mortality. The operating time was 162 (50-300) min. The estimated blood loss was $110(25-1100) \mathrm{ml}$ and two patients received blood transfusion. The postoperative hospital stay was 3.6 $(1-14)$ days. The resected malignancy in 52 patients included metastases in 47 patients (44 colorectal adenocarcinoma, three others) and hepatocellular carcinoma $(\mathrm{n}=5)$, and the $\mathrm{R} 0$ resection rate was $86.6 \%(n=45)$. At $42(6-108)$ months follow-up, $77 \%$ were disease-free, $19 \%$ showed recurrent metastasis (1 hepatic, 4 hepatic and elsewhere, 5 extra-hepatic) and $4 \%$ failed to attend the followup.

Conclusion Our results support the expanding evidence that LLR is safe and efficient for the treatment of benign and malignant liver lesions in carefully selected patients.

Competing interests None declared.

\section{PWE-156 COMMON BILE DUCT STONES; A SURGICAL DISEASE}

doi:10.1136/gutjnl-2012-302514d.156

Z Khanzada, ${ }^{*}$ R Morgan. Department of General Surgery, Glan Clwyd Hospital, Bodelwyddan, UK

Introduction There is no clear consensus, from previous randomised controlled trials and meta-analyses, on the optimum treatment modality for common bile duct (CBD) stones. This study aimed to evaluate the single-stage laparoscopic approach to the management of CBD stones, as treatment of choice in patients who are fit for surgery, and to determine what results can be achieved when this treatment modality is chosen.

Methods The study was performed on a series of patients, both elective and emergency, who underwent laparoscopic CBD exploration, using prospectively collected data. Inclusion criteria were all patients who underwent laparoscopic CBD exploration (along with laparoscopic cholecystectomy) following a positive on-table intraoperative cholangiogram (performed selectively on patients with suspected CBD stones). The rate of successful CBD clearance was measured; markers of unsuccessful CBD clearance included finding of residual stones on $\mathrm{T}$ tube cholangiogram or MRCP, and the need for post-operative ERCP for clearance of either stones known to be left behind at the end of the procedure, or presenting symptomatically in the post-operative period. Morbidity was measured by identifying complications, including both intra-operative and postoperative, by case note review. Hospital stay was recorded from the date of admission to the date of discharge. Mortality was measured from hospital mortality audit records. Outcomes were noted in both elective and emergency situations. Finally a comparison of our experience was made with the recent published data.

Results In a series of 140 patients, successful clearance of CBD stones was achieved in $95.7 \%$ of cases. Retained stones were found in $3.6 \%$ cases and another $3.6 \%$ developed post-operative complications. A post-operative mortality rate of $1.4 \%$ was recorded, which was due to reasons not directly related to the procedure. The most common length of stay was 2 days, although the median length of stay was 4 days. $25 \%$ of cases were done as emergencies and $75 \%$ were elective. Overall conversion rate to open surgery was up to $12.8 \%$, which mainly occurred in the emergency cases.

Conclusion Laparoscopic CBD exploration can be chosen as a safe and effective first line treatment for $\mathrm{CBD}$ stones; better results can be anticipated in elective (compared with emergency) patients.

Competing interests None declared. 